\title{
Le vieil homme et son robot
}

\author{
Kurt Seifert
}

Le film Robot \& Frank traite de la relation entre l'homme et la machine et tourne autour de la question de savoir si le monde virtuel peut remplacer ce dont les hommes ont besoin : des relations qui transmettent un message.

\section{Le contexte}

L'intelligence artificielle conçue et programmée par les hommes se révèlera-t-elle être un savoir-faire humain réfléchi dans un futur proche ? Cette pensée nous occupe depuis longtemps et elle s'exprime également dans les produits de culture de masse. Les espoirs comme les craintes sont liés aux points suivants : l'intelligence artificielle pourrait nous aider à atteindre plus rapidement « le royaume de la liberté » (Karl Marx) en laissant le robot travailler pour nous. Elle pourrait toutefois également entraîner une absolue dépendance au monde que nous avons nous même créé. L'industrie culturelle actuelle crée en masse ce genre d'utopies négatives.

\section{Le film}

La machine comme aidant ou dominant l'homme relève d'un domaine de questions dont le film Robot \& Frank s'approche de manière assez souple et auquel il essaie d'apporter une réponse originale. Frank (joué par le très convaincant Frank Langella), un ancien voleur de bijoux connu dans sa jeunesse, est aujourd'hui un vieil homme assez étourdi. Son fils Hunter (James Marsden) dont on ne sait pas ce qui l'anime, se fait des soucis pour son père et souhaiterait le placer dans un foyer pour personnes âgées. Mais Frank s'obstine même après que son fils le force à prendre un robot assistant semblable à un robot Playmobil de taille humaine. Cel dernier doit l'aider à garder une alimentation saine et à se tenir à un emploi du temps quotidien structuré avec des promenades et du jardinage.

Cela donne alors une parodie légère de toutes sortes de «modèles d'activation » qui sont courants dans les établissements pour personnes âgées. Si Frank ne se laisse pas motiver par son robot par une activité sensée, il prend plaisir d'un autre côté à la présence du nouvel occupant... en le rendant complice de ses plans de cambriolage. Le vieux truand ne peut en effet pas décrocher et il n'a pas encore perdu toute sa mémoire quand il s'agit de réactiver ses anciennes aptitudes. Le robot semble d'abord hésiter, mais ensuite il donne son accord pour devenir un instrument de méfait. Toutefois, il est bien plus qu'un instrument car il semble revêtir des traits humains même s'il insiste à plusieurs reprises sur le fait qu'il n'est pas une personne mais seulement un programme. 
Suite à des recherches informatiques, la police dépiste Frank qui, pour ne pas s'inculper lui-même, doit, le cœur gros (car le robot est devenu son « ami » comme Frank l'avoue à son fils) effacer la «mémoire » de son complice et donc désactiver le robot. L'être artificiel n'est donc ni maître ni valet mais plutôt quelque chose comme un vis-à-vis. Pendant un long moment, l'humanisation de la technologie semble être devenue possible.

\section{Interprétation}

L'histoire du film se déroule dans un « avenir proche » comme inséré au début de l'action. Les événements ne mènent cependant pas à un monde meilleur mais dans un monde qui essaie de remplacer le contact humain par une technologie poussée. Frank, dont les pertes de mémoire semblent surtout se rapporter à sa famille, c'est ainsi qu'il reconnaît dans la bibliothécaire Jennifer (Susan Sarandon, devenue célèbre avec La dernière marche (Dead Man Walking)) avec laquelle il aime bien discuter, que bien plus tard son ancienne femme, donne finalement son accord pour entrer dans un foyer pour personnes âgées où il est déjà d'usage que les résidents soient accompagnés de leur robot. Le film se termine par ce qui semble être un happyend: sa femme, son fils et sa fille Madison (Liv Tyler) sont réunis à table avec Frank, mangent et rient ensemble, mais le regard de l'homme visiblement devenu vieux dit tout autre chose : la relation humaine qu'il a tant désiréee n'est pas vraiment possible avec les membres de sa famille. Et le robot qui semble s'être transformé en partenaire s'est révélé être une partie d'un monde virtuel qui peut disparaître rien qu'en appuyant sur un bouton.

Robot \& Frank n'est pas un film sur le début de la démence mais sur l'obstination d'un vieil homme qui, malgré toute l'autonomie affichée (qui s'émiette toutefois de plus en plus), rêve d'amitié que même les meilleurs soins ne peuvent pas remplacer.

Robot \& Frank (2012), 86 minutes, Senator Home Entertainment. Scénario : Christopher Ford, Réalisation : Jake Schreier.

Auteur : Kurt Seifert est Directeur de Recherche \& Bases chez Pro Senectute Schweiz.

Contact : kurt.seifert@pro-senectute.ch 